\title{
Searching for the Immune Basis of Obsessive-Compulsive Disorder
}

\author{
Antonio Lucio Teixeira ${ }^{a}$ b David Henrique Rodrigues ${ }^{a}$ \\ Andrea Hovarth Marques ${ }^{c}$ Eurípedes C. Miguel ${ }^{c}$ Leonardo F. Fontenelle ${ }^{d}$ \\ a Laboratório Interdisciplinar de Investigação Médica, Faculdade de Medicina, and ${ }^{\mathrm{b}}$ Instituto de Estudos Avançados \\ Transdisciplinares (IEAT), Universidade Federal de Minas Gerais (UFMG), Belo Horizonte, 'Instituto de Psiquiatria, \\ Universidade de São Paulo (USP), São Paulo, and d'Instituto de Psiquiatria, Universidade Federal do Rio de Janeiro \\ (UFRJ), Rio de Janeiro, Brazil
}

\section{Key Words}

Obsessive-compulsive disorder · Anti-basal ganglia

antibodies $\cdot$ Rheumatic chorea

\begin{abstract}
The etiopathogenesis of obsessive-compulsive disorder (OCD) remains elusive. Clinical observation of the elevated frequency of obsessive-compulsive symptoms in patients with rheumatic fever, a post-streptococcal autoimmune disease, prompted the study of immune parameters in OCD. Anti-basal ganglia antibodies have been described in a subset of OCD patients. The assessment of circulating cytokines and immune cells confirmed unequivocal changes in at least some patients, although it is difficult to establish a particular immune profile in OCD. Several factors, including the use of psychotropic drugs and the presence of comorbid conditions, seem to influence these immune parameters.
\end{abstract}

(c) 2014 S. Karger AG, Basel

\section{Introduction}

Obsessive-compulsive disorder (OCD) is characterized by different combinations of obsessive thoughts, images or urges that are associated with anxiety or distress, and may be followed by compulsive mental or motor rituals performed to reduce or neutralize these uncomfortable feelings $[1,2]$. Generally, OCD symptoms tend to organize themselves into four to five different thematic clusters, including contamination/washing, sexual/religious, aggressive/checking, symmetry and ordering, and hoarding themes [3].

Once believed to be a form of anxiety disorder, increasing evidence from a number of disciplines (e.g. clinical sciences, genetics and neuroimaging) has emerged supporting the unique diagnostic status of OCD. Indeed, this distinctiveness has been acknowledged in the most recent edition of the DSM diagnostic system (DSM-5) [4], where OCD is at the core of a new category termed 'obsessive-compulsive and related disorders', together with body dysmorphic disorder, hoarding disorder, trichotillomania and excoriation disorder [5].

Until the 1950's, OCD was considered to be utterly rare, affecting only $0.05 \%$ of the general population according to pioneering German epidemiological studies [6]. However, since the 1980's, modern investigations prompted by the North American Epidemiological Catchment Area, ECA, study have increasingly suggested that OCD is a common condition. OCD is now recognizable among the four most frequent psychiatric conditions, affecting between 1.94 and $3.29 \%$ of individuals across the five ECA sites [7].

\section{KARGER}

E-Mail karger@karger.com

www.karger.com/nim
(C) 2014 S. Karger AG, Basel

$1021-7401 / 14 / 0213-0152 \$ 39.50 / 0$
Antonio L. Teixeira

Laboratório Interdisciplinar de Investigação Médica, Faculdade de Medicina Av. Alfredo Balena, 190, Sala 281

Santa Efigênia, Belo Horizonte 30130-100 (Brazil)

E-Mail altexr@gmail.com 
Despite being quite frequent and often incapacitating, OCD has been less frequently investigated, at least from the pathophysiological standpoint, than other less frequent mental illnesses such as schizophrenia or bipolar disorder. In recent years, great attention has been directed to the role played by the immune system in the pathogenesis of several neuropsychiatric disorders. This paper discusses the evidence on the role of immune dysfunction in OCD.

\section{Overview of OCD Etiopathogenesis}

The available data support a model whereby OCD-related genes confer susceptibility to individuals that, once exposed to certain environmental scenarios, exhibit OCD symptoms [2]. Although somewhat simplistic and potentially applicable to several different mental illnesses, this model provides a framework to test different etiopathogenic hypotheses, including whether other factors may be involved in the pathway towards the emergence of OCD.

Several family and twin studies have provided evidence for heritability of OCD. A multitude of genetic association studies in OCD have been performed in the last 2 decades, but few consistent findings have been achieved. A recent comprehensive meta-analysis examining a total of 230 polymorphisms from 113 genetic-association studies found that OCD was associated with serotoninrelated polymorphisms (5-HTTLPR and HTR2A) and, in males only, with polymorphisms involved with catecholamine modulation (COMT and MAOA) [8]. Secondary analysis comprising polymorphisms that were evaluated in fewer than five data sets identified other candidate polymorphisms that were associated with trophic factors, neurotransmitters and an immunologic factor, i.e. TNFA (rs361525) [8].

To overcome the limitations related to association studies (e.g. variable statistical power) and the intrinsic heterogeneity of OCD, a genome-wide association study, GWAS, has been proposed. The first GWAS study of OCD that analyzed 400 trios, 1,465 cases and 5,557 controls for 469,410 autosomal and 9,657 X-chromosome single-nucleotide polymorphisms (SNPs) was recently published [9]. No SNPs were identified to be associated with OCD at a genome-wide significant level in the combined trio-case-control sample. It is worth mentioning, however, that the top-ranked SNPs from the trio-casecontrol analysis related to transcriptional regulation and glutamatergic neurotransmission and signaling. As with other complex neuropsychiatric disorders, OCD suscep- tibility seems to be determined by the sum of a large number of genes with modest effect, and most of them are related with neurotransmitter systems in OCD.

Neuroimaging studies have demonstrated that OCD is associated with hyperactivation of specific frontostriatal circuits, especially those involving the orbitofrontal cortex and the ventral striatum (component of the basal ganglia) [10]. By affecting these circuits with drugs (e.g. serotonin reuptake inhibitors), cognitive-behavioral therapy, neurosurgery or neurostimulation techniques, it is possible to mitigate OCD symptoms $[1,11]$. Therefore, abnormal modulation of these frontostriatal circuits, for instance, by gene-associated dysfunction in neurotransmitter systems, may confer susceptibility to OCD. Structural lesions (e.g. stroke) compromising frontostriatal circuits can also be followed by obsessive-compulsive phenomena [12]. This seems to be the case in neurological conditions evolving with chorea (random and purposeless involuntary movements), like neuroacanthocytosis and Sydenham's chorea (SC). The neuroacanthocytoses are a group of genetic diseases presenting peripheral blood acanthocytes and progressive pathology in the basal ganglia that account for the characteristic choreic symptoms and elevated rates of OCD [13].

\section{Rheumatic Fever: A Model for Autoimmune OCD}

In the late 1980's, Swedo et al. [14] at the National Institute of Mental Health (NIMH, Bethesda, Md., USA) reported increased frequency of obsessive-compulsive symptoms in children and adolescents with SC. SC is a delayed neurological complication of group $\mathrm{A} \beta$-hemolytic streptococcal (GABHS) pharyngitis and constitutes one of the major criteria of acute rheumatic fever (RF), an inflammatory autoimmune disease affecting mainly the heart valves. In SC, the typical age at onset of motor symptoms is around 9 years old with a female preponderance [15]. The initial findings of higher frequency of obsessivecompulsive symptoms and OCD in SC was not only confirmed, but also extended, in subsequent studies, to patients with present or past history of RF with or without SC [16-18].

Pathological studies performed in the first half of the 20 th century indicated that SC is associated with localized infiltration of mononuclear cells, perivascular cuffs and neuronal loss in the basal ganglia [19]. Later structural and functional neuroimaging studies confirmed basal ganglia compromise in SC [20]. The immune basis of SC has also been defined, and SC fulfills the modified Wi- 
tebsky's criteria for autoimmune disease [21]: (i) the presence of autoreactivity marked by anti-neuronal or antibasal ganglia antibodies (ABGA); (ii) recognition of a specific autoantigen (e.g. tubulin); (iii) induction of the disease in an animal model, and (iv) passive transfer of the disease to the animal $[22,23]$. Molecular mimicry between $N$-acetyl- $\beta$-D-glucosamine, the dominant epitope of the group A streptococcal carbohydrate, and mammalian gangliosides (i.e. lysoganglioside GM1) has been considered the mechanism underlying the autoimmune process in SC $[24,25]$. In this context, the basal ganglia have been implicated as a target of post-streptococcal autoimmune mechanisms in SC.

The presence of psychiatric disorders in patients with a previous history of RF suggests that GABHS may trigger $\mathrm{OCD}$ and related disorders that may persist throughout life regardless of GABHS reinfections. Family studies have suggested that this relationship could be familial based on findings that obsessive-compulsive-related disorders and anxiety disorders, such as generalized anxiety disorders and separation anxiety, aggregate more frequently in first-degree relatives of RF probands when compared with controls $[26,27]$. In the search for candidate genes, two polymorphisms of the promoter region of the tumor necrosis factor- $\alpha$ (TNF- $\alpha$ ) gene have been associated with both OCD and RF, which is an interesting finding as TNF- $\alpha$ is a proinflammatory cytokine involved in $\mathrm{RF}$ and several other autoimmune diseases $[28,29]$. Therefore, it is possible that both obsessive-compulsiverelated disorders and RF share a common genetic vulnerability mediated by genes relevant for immunologic processes.

Based on the association between GABS with obsessive-compulsive spectrum disorders, an autoimmune model for childhood-onset neuropsychiatric disorder has been proposed. Swedo et al. [14] argued that a subset of childhood tic disorders and OCDs could be causally related to streptococcal infection outside the context of RF, the so-called PANDAS, an acronym for 'pediatric autoimmune neuropsychiatric disorders associated with streptococcal infection'.

The concept of PANDAS was challenged by several subsequent studies. For instance, Kurlan et al. [30] have provided reliable information based on an elegant prospective, blinded, case-control study of children who met diagnostic criteria for PANDAS. Their striking finding was that the large majority of psychiatric exacerbations in this subgroup of patients could not be related to GABHS infections, contradicting the hallmark of the PANDAS hypothesis. Their findings suggest that OCD and Tou- rette syndrome are complex and heterogeneous conditions that may include subgroups prone to be triggered by a possible array of precipitants, including GABHS infections. Despite the great controversy associated with the concept of PANDAS, it was relevant to foster the investigation of immune-mediated basal ganglia disorders and also the immune basis of OCD.

\section{ABGA: More Than a Biomarker?}

The spectrum of post-streptococcal autoimmune disorders has been significantly enlarged in the last decade, comprising not only psychiatric syndromes, but also a series of extrapyramidal movement disorders like dystonia, myoclonus, tremor and parkinsonism [31]. The main laboratory evidence supporting this group of disorders comes from the identification of increased titers of ABGA and/or anti-streptolysin O (ASO) antibodies.

In children and adolescents with OCD, a series of studies investigated ABGA and ASO antibodies in the blood, finding inconsistent results with positive and negative findings [32]. More recently, two independent cross-sectional studies with adult OCD patients assessing ABGA and ASO reached similar conclusions. Maina et al. [33] performed a case-control study comprising 74 OCD patients and 44 controls with major depressive episodes, finding a higher percentage of positive ASO titers in OCD subjects than controls (16.3 vs. $2.3 \%$ ), but comparable levels of a series of autoantibodies (anti-nuclear, anti-double stranded DNA, anti-neutrophil cytoplasmic, anti-thyroglobulin, anti-thyroid peroxidase and anti-thyrotropin receptor antibodies). The difference regarding ASO titers could be partially explained by the younger mean age of the OCD patients $(35.3 \pm 12.7)$ in comparison with controls $(56.0 \pm 16.8)$. Interestingly only 4 out 74 OCD patients and none of the controls resulted positive for ABGA. The authors concluded that the majority of adult OCD patients do not seem to have major autoimmune dysfunction, but ABGA are present in a small group of patients and may play a pathogenic role.

Nicholson et al. [34] tested 96 adult OCD patients and 50 controls (33 with chronic depression and 17 with schizophrenia) for the presence of ASO and ABGA. The rates of positivity for ASO in both the OCD and control groups were low and the differences were not significant. Positivity for ABGA was observed in 19/96 (19.8\%) participants with OCD compared with $2 / 50$ (4\%) controls $(\mathrm{p}=0.012)$. The majority of positive OCD sera $(13 / 19)$ 
had antibodies against the enolase antigen. They also concluded that antibody-mediated autoimmunity might play a pathogenic role in a subset of OCD patients.

To further investigate the role of ABGA in OCD, Bhattacharyya et al. [35] examined ABGA in the serum and cerebrospinal fluid (CSF), and measured CSF amino acids (GABA, glutamate, glycine and taurine) in 23 drugnaïve adult $O C D$ patients compared with 23 healthy controls. There was no significant difference in serum autoantibodies binding to basal ganglia homogenates (as assessed by Western blot), but there was more binding of CSF autoantibodies in OCD patients in contrast with controls, possibly indicating intrathecal ABGA synthesis. CSF glutamate and glycine levels were also significantly higher in OCD subjects compared with controls. Interestingly, CSF glycine levels were significantly increased in OCD patients with CSF ABGA, suggesting that the abnormal levels of excitatory neurotransmitters reported in OCD might be related to ABGA. In sum, these ABGA in OCD could be: (i) biomarkers of an autoimmune process (related or not to streptococcal infection); (ii) produced as a result of neuronal damage, or (iii) represent an epiphenomenon. Although it has been largely accepted that ABGA may support an immune basis for at least some OCD patients, further studies are warranted to demonstrate their pathogenic role.

\section{Infectious Diseases and OCD}

As mentioned above, in addition to GABHS, other infectious diseases may be of paramount importance in the development of OCD. Toxoplasma gondii is a ubiquitous parasite that has already been implicated in the pathogenesis of schizophrenia [36]. This is mainly supported by serological studies showing higher prevalence of antiToxoplasma IgG antibodies in schizophrenic patients in comparison with controls. As the parasite has a strong tropism for the central nervous system, with preferential localization in the basal ganglia, Miman et al. [37] investigated the seropositivity rate for anti-Toxoplasma IgG antibodies in 42 OCD patients and 100 controls. The seropositivity rate was significantly higher in OCD patients $(47.6 \%)$ than in controls $(19 \%$; $p<0.01)$, suggesting a role for chronic toxoplasmosis in OCD pathogenesis. The mechanisms by which $T$. gondii affects neural functions are uncertain, but may involve direct neuronal and glial damage, changes in neurotransmitter pathways and induction of immune response with the release of several cytokines [36].

Searching for the Immune Basis of OCD
Borna disease virus is an RNA virus that primarily determines neurological syndromes, including extrapyramidal symptoms, in higher vertebrates like horses, sheep and cattle. In the early 1990's Borna disease virus was associated with psychiatric disorders, mainly mood disorders. Dietrich et al. [38] reported an association between Borna disease virus-specific circulating immune complexes and dysfunction in the cingulate cortex of OCD patients submitted to a mental flexibility test. It was suggested that Borna disease virus-induced immune response would be responsible for enhanced levels of glutamate, leading to thalamocortical dysfunction [39].

Therefore, the immune system seems to play a pivotal role in OCD cases associated either with the autoimmunity triggered by $\beta$-hemolytic streptococcal infection, or with T. gondii or Borna disease virus infections. Based on these unexpected but interesting associations, several studies have assessed immune parameters in OCD.

\section{Changes in Immune Parameters in OCD}

A series of studies has investigated the levels of circulating cytokines in OCD patients in comparison with control subjects, with mixed results [32]. To overcome this, a meta-analysis was performed by Gray and Bloch [40], concluding that IL-1 $\beta$ is decreased in patients with OCD, while no difference was found in IL- 6 and TNF- $\alpha$ levels. In stratified subgroup analysis, moderating effects of age and inclusion of patients under pharmacological treatment and with comorbid mood disorders were reported. For instance, IL-6 levels were significantly increased in drug-free OCD adults, while IL-6 levels were significantly lower in children with OCD who were taking psychotropic drugs [40].

The low number of studies assessing ex vivo cytokine production has prevented a meta-analysis of these data. These limited studies suggested that ex vivo IL- 6 production may be reduced, with no significant changes in the production of IL- $1 \beta$ and TNF- $\alpha$ [40]. It is worth emphasizing that this reduction in inflammatory cytokines, indicating a noninflammatory profile in OCD, is in the opposite direction to that reported for other major psychiatric disorders, like schizophrenia and bipolar disorder. This decrease in IL-1 $\beta$ also deserves a more thorough investigation as this cytokine may play a role in central nervous system repair, especially in remyelination [41], and OCD has been associated with several structural changes, including in the corpus callosum [42].

Neuroimmunomodulation 2014;21:152-158 DOI: 10.1159/000356554 
We performed the largest study to date evaluating cytokines, chemokines and other inflammatory mediators in adult patients with OCD. In comparison with healthy controls, patients with OCD exhibited increased plasma levels of soluble receptors of TNF- $\alpha$ type 1 (sTNFR1) and type 2 (sTNFR2), and the chemokines CXCL8/IL- 8 and CCL3/MIP-1 $\alpha$ [43]. The discrepancy between the studies may be ascribed to sample differences, including the age of patients, disease duration and severity, and use of psychotropic drugs. Increased circulating levels of sTNFR1 and sTNFR2 in the absence of increased TNF- $\alpha$ levels has already been reported and can be tentatively explained by TNF- $\alpha$ molecule instability and its consequent short halflife. The assessment of sTNFR1 and sTNFR2 was reported to be reliable surrogate markers of the overall production and activity of TNF- $\alpha$ [44]. Interestingly, plasma levels of sTNFR1 correlated positively with the severity of washing symptoms in our OCD sample. This could indicate that certain subsets of OCD patients would be more associated with immune dysfunction than others. It remains to be determined whether these patients are the same OCD group with increased circulating levels of ABGA. We hypothesized that increased sTNFR1-related washing symptoms might represent some sort of behavioral correlate to deal with infectious agents [43]. Although this hypothesis is highly speculative, Murphy and Pichichero [45] have already reported that $75 \%$ of patients diagnosed with PANDAS had germ- or illness-related obsessions, causing compulsive hand washing or excessive toilet hygiene rituals.

Only two studies have investigated CSF levels of cytokines in OCD. Mittleman et al. [46] compared CSF levels of IL-2, IL-4, IL-5, IL-10, TNF- $\alpha$, IFN- $\gamma$ and lymphotoxin among three groups of childhood psychiatric disorders, OCD, schizophrenia and attention-deficit/hyperactivity disorder, with no healthy control group. They found a relative skewing of CSF cytokines toward a Th1 profile in patients with OCD, and a relative preponderance of Th2 profile in schizophrenia. Carpenter et al. [47] did not find significant difference in CSF levels of IL- 6 between adult OCD patients and matched healthy controls.

Studies assessing immune cell phenotype in OCD are also scarce [32]. Marazziti et al. [48, 49] reported that OCD patients showed a significant increase of CD8+ and decrease of CD4+ T lymphocytes when compared with controls. Interestingly, these immunophenotypic changes were reverted by treatment with selective serotonin reuptake inhibitors, in parallel with clinical improvement [48]. Therefore, the alterations of immune cells should be considered a state-dependent marker, perhaps related to stress [48].

Stress modulates immune function through the activation of the hypothalamic-pituitary-adrenal axis and the consequent release of glucocorticoids that exert immunosuppressive effects. This could partly explain the reduced ex vivo production and circulation of proinflammatory cytokines [40]. The presence of comorbid conditions, especially major depression, and the use of psychotropic drugs are also factors that influence immune activity and could contribute to the contradictory findings described in the literature. However, the real mechanisms underlying the immune imbalance in psychiatric disorders, including OCD, remain to be determined.

\section{Concluding Remarks}

OCD is a chronic and sometimes incapacitating neuropsychiatric disorder with a complex etiopathogenesis. The observation of a higher frequency of OCD in patients with RF and its precipitance with infectious diseases prompted the study of immune parameters in the disease. Although the results to date are difficult to interpret as no discrete immune profile has emerged, there are unequivocal immune changes in at least some OCD patients. Future challenges include: (i) defining whether these immune changes are trait or state dependent, or secondary to the response of disease burden and their potential to be regarded as biomarkers; (ii) identifying the OCD subtypes more associated with immune physiopathology (possibly some cases of early onset - with contamination/ washing symptoms?); (iii) testing the efficacy of immunebased strategies in eligible subgroups of OCD.

\section{Acknowledgments}

This work was partly funded by CNPq and Fapemig.

References
Teixeira/Rodrigues/Marques/Miguel/ Fontenelle 
-4 Stein DJ, Fineberg NA, Bienvenu OJ, Denys D, Lochner C, Nestadt G, Leckman JF, Rauch SL, Phillips KA: Should OCD be classified as an anxiety disorder in DSM-V? Depress Anxiety 2010;27:495-506.

5 American Psychiatric Association: Diagnostic and Statistical Manual of Mental Disorders: DSM-5. Washington, American Psychiatric Association, 2013.

6 Rudin E: On the problem of compulsive disease with special reference to its hereditary relations (in undetermined language). Arch Psychiatr Nervenkr Z Gesamte Neurol Psychiatr 1953;191:14-54.

7 Fontenelle LF, Hasler G: The analytical epidemiology of obsessive-compulsive disorder: risk factors and correlates. Prog Neuropsychopharmacol Biol Psychiatry 2008;32:1-15.

8 Taylor S: Molecular genetics of obsessivecompulsive disorder: a comprehensive metaanalysis of genetic association studies. Mol Psychiatry 2013;18:799-805.

-9 Stewart SE, Yu D, Scharf JM, Neale BM, Fagerness JA, Mathews CA, et al: Genomewide association study of obsessive-compulsive disorder. Mol Psychiatry 2013;18:788798.

10 Harrison BJ, Soriano-Mas C, Pujol J, Ortiz H, Lopez-Sola M, Hernandez-Ribas R, Deus J, Alonso P, Yucel M, Pantelis C, Menchon JM, Cardoner N: Altered corticostriatal functional connectivity in obsessive-compulsive disorder. Arch Gen Psychiatry 2009;66:11891200.

11 Greenberg BD, Rauch SL, Haber SN: Invasive circuitry-based neurotherapeutics: stereotactic ablation and deep brain stimulation for OCD. Neuropsychopharmacology 2010;35: 317-336.

12 Fontenelle LF, Harrison BJ, Yücel M, Pujol J, Fujiwara H, Pantelis C: Is there evidence of brain white-matter abnormalities in obsessive-compulsive disorder? A narrative review. Top Magn Reson Imaging 2009;20:291-298.

13 Walterfang M, Evans A, Looi JC, Jung HH, Danek A, Walker RH, Velakoulis D: The neuropsychiatry of neuroacanthocytosis syndromes. Neurosci Biobehav Rev 2011;35: 1275-1283.

14 Swedo SE, Rapoport JL, Cheslow DL, Leonard HL, Ayoub EM, Hosier DM, Wald ER: High prevalence of obsessive-compulsive symptoms in patients with Sydenham's chorea. Am J Psychiatry 1989;146:246-249.

-15 Teixeira AL, Maia DP, Cardoso F: UFMG Sydenham's chorea rating scale (USCRS): reliability and consistency. Mov Disord 2005; 20:585-591.

-16 Alvarenga PG, Hounie AG, Mercadante MT, Diniz JB, Salem M, Spina G, Miguel EC: Obsessive-compulsive symptoms in heart disease patients with and without history of rheumatic fever. J Neuropsychiatry Clin Neurosci 2006; 18:405-408.
17 Hounie AG, Pauls DL, Mercadante MT, Rosário-Campos MC, Shavitt RG, de Mathis MA, de Alvarenga PG, Cúri M, Miguel EC: Obsessive-compulsive spectrum disorders in rheumatic fever with and without Sydenham's chorea. J Clin Psychiatry 2004;65:994999.

-18 Alvarenga PG, Floresi AC, Torres AR, Hounie AG, Fossaluza V, Gentil AF, Pereira CA, Miguel EC: Higher prevalence of obsessivecompulsive spectrum disorders in rheumatic fever. Gen Hosp Psychiatry 2009;31:178-180.

19 Colony HS, Malamud N: Sydenham's chorea: a clinicopathologic study. Neurology 1956;6: 672-676.

20 Giedd JN, Rapoport JL, Kruesi MJ, Parker C, Schapiro MB, Allen AJ, Leonard HL, Kaysen D, Dickstein DP, Marsh WL, et al: Sydenham's chorea: magnetic resonance imaging of the basal ganglia. Neurology 1995;45:21992202.

21 Rose NR, Bona C: Defining criteria for autoimmune diseases (Witebsky's postulates revisited). Immunol Today 1993;14:426-430.

22 Brimberg L, Benhar I, Mascaro-Blanco A, Alvarez $\mathrm{K}$, Lotan $\mathrm{D}$, Winter C, Klein J, Moses AE, Somnier FE, Leckman JF, Swedo SE, Cunningham MW, Joel D: Behavioral, pharmacological, and immunological abnormalities after streptococcal exposure: a novel rat model of Sydenham chorea and related neuropsychiatric disorders. Neuropsychopharmacology 2012;37:2076-2087.

-23 Teixeira AL, Guimarães MM, Romano-Silva MA, Cardoso F: Serum from Sydenham's chorea patients modifies intracellular calcium levels in PC12 cells by a complement-independent mechanism. Mov Disord 2005;20: 843-845.

24 Kirvan CA, Swedo SE, Heuser JS, Cunningham MW: Mimicry and autoantibody-mediated neuronal cell signaling in Sydenham chorea. Nat Med 2003;9:914-920.

25 Kirvan CA, Cox CJ, Swedo SE, Cunningham MW: Tubulin is a neuronal target of autoantibodies in Sydenham's chorea. J Immunol 2007;178:7412-7421.

-26 Hounie AG, Pauls DL, do Rosario-Campos MC, Mercadante MT, Diniz JB, De Mathis MA, De Mathis ME, Chacon P, Shavitt RG, Curi M, Guilherme L, Miguel EC: Obsessivecompulsive spectrum disorders and rheumatic fever: a family study. Biol Psychiatry 2007; 61:266-272.

27 Seixas AA, Hounie AG, Fossaluza V, Curi M, Alvarenga PG, De Mathis MA, De Mathis ME, Vallada H, Pauls D, de Bragança Pereira CA, Miguel EC: Anxiety disorders and rheumatic fever: is there an association? CNS Spectr 2008; 13:1039-1046.

28 Ramasawmy R, Faé KC, Spina G, Victora GD, Tanaka AC, Palácios SA, Hounie AG, Miguel EC, Oshiro SE, Goldberg AC, Kalil J, Guilherme L: Association of polymorphisms within the promoter region of the tumor necrosis factor- $\alpha$ with clinical outcomes of rheumatic fever. Mol Immunol 2007;44:1873-1878.
29 Hounie AG, Cappi C, Cordeiro Q, Sampaio AS, Moraes I, Rosário MC, Palácios SA, Goldberg AC, Vallada HP, Machado-Lima A, Nakano E, Kalil J, Pauls D, Pereira CA, Guilherme L, Miguel EC: TNF- $\alpha$ polymorphisms are associated with obsessive-compulsive disorder. Neurosci Lett 2008;442:86-90.

30 Kurlan R, Johnson D, Kaplan EL, Tourette Syndrome Study Group: Streptococcal infection and exacerbations of childhood tics and obsessive-compulsive symptoms: a prospective blinded cohort study. Pediatrics 2008; 121:1188-1197.

31 Dale RC: Immune-mediated extrapyramidal movement disorders, including Sydenham chorea. Handb Clin Neurol 2013;112:12351241.

32 Rocha FF, Correa H, Teixeira AL: Obsessivecompulsive disorder and immunology: a review. Prog Neuropsychopharmacol Biol Psychiatry 2008;32:1139-1146.

33 Maina G, Albert U, Bogetto F, Borghese C, Berro AC, Mutani R, Rossi F, Vigliani MC: Anti-brain antibodies in adult patients with obsessive-compulsive disorder. J Affect Disord 2009;116:192-200.

- 34 Nicholson TR, Ferdinando S, Krishnaiah RB, Anhoury S, Lennox BR, Mataix-Cols D, Cleare A, Veale DM, Drummond LM, Fineberg NA, Church AJ, Giovannoni G, Heyman I: Prevalence of anti-basal ganglia antibodies in adult obsessive-compulsive disorder: cross-sectional study. Br J Psychiatry 2012; 200:381-386.

35 Bhattacharyya S, Khanna S, Chakrabarty K, Mahadevan A, Christopher R, Shankar SK: Anti-brain autoantibodies and altered excitatory neurotransmitters in obsessive-compulsive disorder. Neuropsychopharmacology 2009;34:2489-2496.

36 Fabiani S, Pinto B, Bruschi F: Toxoplasmosis and neuropsychiatric diseases: can serological studies establish a clear relationship? Neurol Sci 2013;34:417-425.

37 Miman O, Mutlu EA, Ozcan O, Atambay M, Karlidag R, Unal S: Is there any role of Toxoplasma gondii in the etiology of obsessivecompulsive disorder? Psychiatry Res 2010; 177:263-265.

38 Dietrich DE, Zhang Y, Bode L, Münte TF, Hauser U, Schmorl P, Richter-Witte C, Gödecke-Koch T, Feutl S, Schramm J, Ludwig H, Johannes S, Emrich HM: Brain potential amplitude varies as a function of Borna disease virus-specific immune complexes in obsessive-compulsive disorder. Mol Psychiatry 2005;10:519-520.

39 Rotge JY, Aouizerate B, Tignol J, Bioulac B, Burbaud P, Guehl D: The glutamate-based genetic immune hypothesis in obsessive-compulsive disorder: an integrative approach from genes to symptoms. Neuroscience 2010; 165:408-417. 
40 Gray SM, Bloch MH: Systematic review of proinflammatory cytokines in obsessivecompulsive disorder. Curr Psychiatry Rep 2012;14:220-228.

-41 Mason JL, Suzuki K, Chaplin DD, Matsushima GK: Interleukin- $1 \beta$ promotes repair of the CNS. J Neurosci 2001;21:7046-7052.

42 Lopez KC, Lalonde F, Mattai A, Wade B, Clasen L, Rapoport J, Giedd JN: Quantitative morphology of the corpus callosum in obsessive-compulsive disorder. Psychiatry Res 2013;212:1-6.

43 Fontenelle LF, Barbosa IG, Luna JV, de Sousa LP, Abreu MN, Teixeira AL: A cytokine study of adult patients with obsessive-compulsive disorder. Compr Psychiatry 2012;53:797804 .
44 Scalzo P, Kummer A, Cardoso F, Teixeira AL: Increased serum levels of soluble tumor necrosis factor- $\alpha$ receptor- 1 in patients with Parkinson's disease. J Neuroimmunol 2009; 216:122-125.

45 Murphy ML, Pichichero ME: Prospective identification and treatment of children with pediatric autoimmune neuropsychiatric disorder associated with group A streptococcal infection (PANDAS). Arch Pediatr Adolesc Med 2002;156:356-361.

- 46 Mittleman BB, Castellanos FX, Jacobsen LK, Rapoport JL, Swedo SE, Shearer GM: Cerebrospinal fluid cytokines in pediatric neuropsychiatric disease. J Immunol 1997;159: 2994-2999.

-47 Carpenter LL, Heninger GR, McDougle CJ, Tyrka AR, Epperson CN, Price LH: Cerebrospinal fluid interleukin-6 in obsessive-compulsive disorder and trichotillomania. Psychiatry Res 2002;112:257-262.
8 Marazziti D, Mungai F, Masala I, Baroni S, Vivarelli L, Ambrogi F, Catena Dell'Osso M, Consoli G, Massimetti G, Dell'Osso L: Normalisation of immune cell imbalance after pharmacological treatments of patients suffering from obsessive-compulsive disorder. J Psychopharmacol 2009;23:567-573.

49 Marazziti D, Presta S, Pfanner C, Gemignani A, Rossi A, Sbrana S, Rocchi V, Ambrogi F, Cassano GB: Immunological alterations in adult obsessive-compulsive disorder. Biol Psychiatry 1999;46:810-814. 